\title{
Review on Reported Concussion, Identification and Management in Extreme Sports
}

\author{
C. N. Seehusen ${ }^{1}$, V. Mucci ${ }^{2,4}$, K. E. Welman ${ }^{3}$, C. J. Browne ${ }^{4}$, F. Feletti ${ }^{5,6}$, A. J. Provance ${ }^{1,7}$ \\ 1 Sports Medicine Center, Children's Hospital Colorado, Aurora, Colorado, USA \\ 2 Laboratory of Neuromotor Physiology, IRCCS Santa Lucia Foundation, Rome, Italy \\ 3 Sport Science Department, Movement Laboratory, Faculty of Medicine and Health Sciences, Stellenbosch \\ University, South Africa \\ 4 School of Science, Western Sydney University, New South Wales, Australia \\ 5 Department of Diagnostic Imaging Society, Ausl Romagna, S. Maria delle Croci Hospital, Ravenna, Italy \\ ${ }^{6}$ Department of Electronics Information and Bioengineering, Politecnico di Milano University, Milan, Italy \\ 7 Department of Orthopedics, University of Colorado School of Medicine, Aurora, Colorado, USA
}

\section{CORRESPONDING AUTHOR:}

Aaron J. Provance

Sports Medicine Center

Children's Hospital Colorado

Aurora, Colorado, USA

E-mail: Aaron.provance@childrenscolorado.org

DOI:

10.32098/mltj.02.2020.14

LEVEL OF EVIDENCE: 3a

\begin{abstract}
SUMMARY
Background. As participation in extreme sports continues to grow internationally, the number of concussions sustained during these activities is predicted to increase. Due to the lack of organizational frameworks, governing rules, or regulated competitive structures, the incidence of concussion and its management specific to extreme sports athletes remains difficult to determine.

Methods. Relevant papers were screened from PubMed using a combination of terms related to extreme sports and concussion. After considering existing literature, papers that did not fit the authors' agreed-upon definition of extreme sports were excluded.

Results. Eleven manuscripts met inclusion criteria. Of the eleven, only five studies reported on more than one extreme sport while the other six were sport-specific. Three were review papers that used sport-specific data to generalize about extreme sports. Conclusions. The results of our review indicate that the current literature available for concussion in extreme sports varies highly in study design and type of sports investigated. Due to the lack of knowledge regarding concussions in extreme sports, there needs to be an emphasis to better document and record concussion incidence in extreme sports, as well as the need to develop specific return-to-play guidelines for healthcare professionals treating extreme sports athletes.
\end{abstract}

\section{KEY WORDS}

Concussion; extreme sports medicine; mild-traumatic brain injury; sports-related concussion; post-concussive syndrome.

\section{BACKGROUND}

Concussion is a form of mild Traumatic Brain Injury (mTBI), defined as a complex pathophysiologic process induced by direct or indirect impulsive forces to the head that disrupts the brain's functions (1). Due to the deficiency of normative data for clinical tests, the absence of well-established validated biomarkers, the highly variable post-injury symptom presentation, and the lack of uniformity regarding concussion definition, concussions remain one of the most complex injuries in sports medicine to diagnose and subsequently manage (2-4). This complexity can result in difficulty in understanding the recovery process following injury, and may factor into why athletes are developing persistent post-concussion symptoms $(3,5,6)$.

Sports-Related Concussion (SRC) has become a public health problem, with an estimated 1.6 to 3.8 million SRCs each year in the United States (7). In a recent multicenter cross-sectional study, $30.5 \%$ of athletes reported a previ- 
ously undiagnosed concussion (8). Furthermore, many concussions that occur may go unrecognized or undiagnosed, leading to a potentially greater prevalence of injury. In the same multicenter study, the athletes who reported an undiagnosed concussion had higher mean Post Concussion Symptom Scale (PCSS) score and were more likely to have lost consciousness with their current injury than athletes without previously undiagnosed concussions (8). For athletes in extreme sports in particular, the prevalence and incidence of concussion has not been universally established. Some hypothesize that these athletes may be more vulnerable to sustaining a concussion due to the high forces and accelerations that occur during extreme sports, but an overview of extreme sports and brain injuries has yet to be investigated. Despite this lack in normative data across all extreme sports, there is some data on the subject. According to one recent study, concussions account for $9.4 \%$ of all injuries that reported to emergency departments in X games competitions from 2000-2011 (9). Overall, however, the epidemiological data on injuries in extreme sports are scarce (10).

The term "extreme sports" has become a widely used term to describe a variety of non-traditional sporting activities (11). Terms like "action sports" or "adventure sports" are often used as synonyms $(12,13)$. Specifically, the definition of extreme sports encompasses a wide range of sport activities, which stem from the creative exploration of novel movement experiences and include water-sports (e.g. kitesurfing, SCUBA diving), air-sports (e.g. skydiving, paragliding) and land-sports (e.g. mountain biking, skateboarding, climbing, snowboarding, parkour). In contrast to public opinion, extreme sports cannot be considered as 'high-risk sports', and are not more dangerous than traditional sports, according to existing literature (14). Despite this finding, extreme sports often exploit an external source of energy, such as gravity in BASE (building, antenna, span, and earth) jumping and skydiving, or natural phenomena, such as thermal updrafts and ocean swells in paragliding and surfing, respectively. Under these circumstances, a greater prevalence of high-energy trauma cases may result from mismanaged execution during extreme sports participation (15). If applied directly or indirectly to the head, these high-energy forces may result in concussion or a more severe TBI.

The participation rate in extreme sports has grown exponentially over the last few decades, often surpassing the growth rates of many traditional sporting activities especially among children and adolescents (16-18). This growing interest has been shown by, and accredited to, the creation and increasing popularity of the X Games, the introduction of new events into the Winter Olympics, and nationally televised extreme sports events over the past decade, as described by epidemiological studies (9). Despite its expansive growth in participation rates among all ages, limited data regarding concussion prevalence exist for extreme sports. This deficiency may be attributed to the lack of organizational frameworks and regulated competitive structures during extreme sports relative to other 'traditional' sports (e.g. football, soccer, hockey) $(19,20)$. As the concern for concussion and international participation in extreme sports continues to rise, a structured assessment and return to play protocol needs to be created and considered for this unique subset of athletes $(19,20)$.

We are unaware of any published reviews on concussion incidence and management in extreme sports athletes. Therefore, the purpose of this scoping review is to examine existing studies that have assessed concussion incidence, prevalence, diagnosis, management and outcomes among extreme sports athletes. This study aims to identify gaps in the literature relating to concussion and extreme sports, in order to determine what future research is needed to improve current clinical management of brain injuries in extreme sports athletes.

\section{METHODS}

A PubMed query was completed using the key words "Extreme sport", "Extreme environment", "Extreme conditions", "Action sport" and "Adventure sport", resulting in more than 5800 papers. These results were then filtered to only include those that included patients who sustained a concussion or other head injury (e.g. mTBI, blunt head trauma). This resulted in a total of 11 papers. Of these 11, two were excluded as they did not fit the agreed upon definition of extreme sports, designed by the authors after considering existing literature: "Extreme sport is a competitive, either by comparison or self/peer evaluation, physical activity in which the participant is subjected to unusual physical and mental challenges while being exposed to environmental variables, with a risk of severe injury or fatality in the case of mismanaged execution" $(14,20-22)$. An additional paper was also removed from the analysis because the authors did not provide a breakdown of head injuries that occurred in extreme sports specifically as compared to what was described as "other risk-taking behaviors". Papers were also chosen using the ancestry approach in order to include anecdotal but relevant reports. This resulted in the addition of three additional pertinent papers. As a result, we reviewed 11 total studies. From each study, we extracted its design, period, number of subjects, the proportion of men and women, setting, and the rate of injuries (if applicable). 


\section{RESULTS}

Of the 11 papers, only five reported on more than one extreme sport. Two of the five were reviews on the current literature, using sport-specific papers to provide insight into extreme sports literature as a whole, while the remaining three reported injury location and severity for a specific cohort. The other six papers were sport-specific, four of which were motocross-focused. Of the four motocross papers, one was a review. Most of the sport-specific papers reported injury occurrence classification except for one by Miller et al. which reflected athlete perception of their injuries and management of concussions (23). Additionally, each of the seven papers that described injury rates used data provided by emergency department visits (all but Miller et al. (23)). During analysis one study not only looked at 10 different sports, but also further divided the injuries into three tiers of risk-taking categories: extreme sports with high-risk practice, potential extreme sport and indeterminate risk, and potential extreme sports but low risk (15). In all the papers mentioned in this review, none provided a breakdown of hours of athletic exposure and how that would relate to injury occurrence rates. This breakdown could be useful for indicating if these athletes were pursuing the sport in question recreationally or competitively.

\section{DISCUSSION}

The results of our review indicate that the current literature available for concussion in extreme sports varies highly in study design and type of sports investigated. There are a few studies that report concussion occurrence rates among extreme sports athletes, which have been summarized in table I. Up to now, this review is the only one of which we are aware of uniting different studies and presenting concussion in extreme sports. Of the papers identified that discussed injury occurrence rates, many separated the analysis by the anatomical location (e.g. head, neck, chest, upper and lower extremity) instead of by the type of injury (e.g. concussion, laceration, contusion, fracture etc.) (15,24-26). As a result, there is a lack of concise data regarding rates of concussions alone. Furthermore, existing studies report primarily on emergency department visits and are not reflective of total injuries seen by all physicians who treat extreme sports athletes (e.g. sports medicine providers) $(9,15,24-$ 27). Within emergency departments, providers typically use templates to make the most efficient use of time. Many of these templates include specific traditional sports when assessing sport related injuries, but often use the term "other" to indicate extreme sports. As a result, if there is no written information specifically input by the health care providers idenitifying the extreme sport the data for that specific type of sport is lost (27). Additionally, of those that report on more than one extreme sport, the main focus lies on injury distribution and less on reporting current management and guidelines for return to play protocols.

\section{Epidemiology}

The majority of studies to date regarding concussions and extreme sports are sport-specific (e.g. snowboarding). In a prospective study, Nakaguchi et al. reported that $26 \%$ of all snowboard and $21 \%$ of all ski-related injuries were head injuries (28). That being said, there was no further classification to determine if these head injuries were concussions, fractures, lacerations, etc. Additionally, the data was collected from a trauma center near the most popular skiing areas in Japan, from which, it was impossible to separate those who were extreme sports athletes from those who were recreational skiers/snowboarders (28). In a separate study that reported solely on extreme sports athletes, concussion accounted for $43.9 \%$ of snowboarding injuries and $41.9 \%$ of skiing injuries (9). In an epidemiological study performed in Canada on 1332 reported snowblading, skiing and snowboarding injuries, it was observed that snowboarding related head injuries had a strikingly high incidence in beginners and during backward falling and jumping. In particular, most head injuries occurred during jumping when the surface of the ground was covered with icy packed snow (26). However, a high rate was also observed on well-marked slopes $(29,30)$. The data concluded that snow SRCs are common and that health care professionals assessing these athletes should have increased suspicion of head injuries after a fall or collision. Educating snow sports athletes about the signs and symptoms of concussion should be emphasized to minimize under recognition and underreporting. Also, increased knowledge of concussion in these sports should include primary care providers who may be the initial contact with these patients. When considering other sports such as mountain biking, one study observed that among 494 mountain-biking injuries, concussion represented $4.6 \%(n=23)$ of all injuries (16). This rate of injury appeared to be lower than snowboarding, although the different design of the studies makes a direct comparison impossible. In a different study, concussion accounted for $29.7 \%$ of mountain biking injuries (9), thus showing the current lack of reproducibility of current concussion research in extreme sports. Mountain biking injury data may be misleading due to the lack of health care professionals located at various races or recreational rides. However, a national injury database for high school mountain biking incidents was recently implemented in the U.S., which may lead to more comprehensive and comparable data sets in the future. 
Table I. Studies that investigated extreme sport athletes who sustained head injuries discovered by our PubMed query with the exclusion of reviews. TBI = Traumatic Brain Injury, LOC = Loss of Consciousness, CT = Computed Tomography, ED = Emergency Department, ES = Extreme Sport, ATV = All-Terrain Vehicle, BASE = Building, Antenna, Span and Earth.

\begin{tabular}{llll}
\hline Study & Design & $\begin{array}{l}\text { Study } \\
\text { Period }\end{array}$ & Sample (n) \\
\hline Weber et & Prospective & Jan 2002- & n=278 individuals \\
al., 2018 & & Dec 2012 & $\begin{array}{l}\text { with major injuries } \\
\text { during extreme }\end{array}$ \\
& & & or contact sports \\
& & (extreme sport only \\
& & breakdown: airborne \\
& & & n=105, climbing n=35, \\
& & & skateboarding/skating \\
& & & \\
& & &
\end{tabular}

Gender Aim/Purpose of Rate of Injuries (M) Study

Airborne: Sport specific Head and face injury: 84.8\% injury patterns $\quad 38.5 \%$ Airborne Climbing: and mechanisms, $\quad 40 \%$ Climbing $71.4 \%$ to characterize $\quad 49 \%$ Skating Skating: individuals at risk $\quad 48 \%$ Contact Sports $58.5 \%$ and to identify possible approaches for prevention

Concussions: 9.5\% Airborne $14.3 \%$ Climbing $15.4 \%$ Skating injuries

Gosteli et Retrospective Jan 1998al., 2016 case-series Dec 2008 $\mathrm{n}=616$ injuries $(219$

extreme sports with high risk practice at time of injury, 69 potential ES but low risk at time of fall, 328 ES with indeterminate risk at time of fall)

\begin{tabular}{|c|c|c|c|c|}
\hline $\begin{array}{l}\text { Sharma et } \\
\text { al., } 2013\end{array}$ & $\begin{array}{l}\text { Descriptive } \\
\text { epidemiological } \\
\text { study }\end{array}$ & $2000-2011$ & $\begin{array}{l}\mathrm{n}=4,083,691 \text { injuries }(\mathrm{n} \\
=381,760 \text { head injuries, } \\
9.3 \%)\end{array}$ & $\begin{array}{l}\text { Not } \\
\text { specified }\end{array}$ \\
\hline
\end{tabular}

$70.9 \%$

\section{Describe epidemiology of} extreme sports injuries in adults ( $>15$ years) that required helicopter emergency medical services

Report neck and
head injuries in 7 extreme sports (snowboarding, snowmobiling, surfing, mountain biking, motocross, skateboarding, and snow skiing) at the Winter and Summer $\mathrm{X}$ games via national (U.S.) surveillance of emergency room visits
High risk events: $9.1 \%$ Severe TBI, $6.9 \%$ mTBI; Snowboarding and mountain biking were specifically associated with TBI.

\begin{tabular}{|c|c|c|c|c|c|c|}
\hline $\begin{array}{l}\text { Soreide et } \\
\text { al., } 2007\end{array}$ & $\begin{array}{l}\text { Retrospective } \\
\text { Cohort }\end{array}$ & $1995-2005$ & $\mathrm{n}=20,850$ jumps & $91 \%$ & $\begin{array}{l}\text { Report frequency of } \\
\text { injuries and deaths } \\
\text { in BASE jumping } \\
\text { (Kjerag, Norway) }\end{array}$ & $\begin{array}{l}\text { Injuries occurrence: } \\
9.8 \text { per } 2,500 \text { jumps; } \\
\text { (including ankle } \\
\text { sprains/fracture, minor } \\
\text { head concussion, or a } \\
\text { bruised knee) }\end{array}$ \\
\hline $\begin{array}{l}\text { Moroney } \\
\text { et al., } 2003\end{array}$ & $\begin{array}{l}\text { Prospective } \\
\text { Cohort }\end{array}$ & $\begin{array}{l}\text { April 1999- } \\
\text { April } 2000\end{array}$ & $\begin{array}{l}\mathrm{n}=32 \text { ( } \mathrm{n}=6 \text { with previous } \\
\text { ATV experience) }\end{array}$ & $71.9 \%$ & $\begin{array}{l}\text { Injury prevalence } \\
\text { in ATV-related } \\
\text { accidents }\end{array}$ & Concussions: $6 \%(n=2)$ \\
\hline $\begin{array}{l}\text { Larson et } \\
\text { al., } 2009\end{array}$ & $\begin{array}{l}\text { Retrospective } \\
\text { Case-series }\end{array}$ & $2000-2007$ & $\begin{array}{l}\mathrm{n}=249 \text { individuals }(299 \\
\text { separate injury episodes) }\end{array}$ & $93.2 \%$ & $\begin{array}{l}\text { Injury distribution } \\
\text { and severity in } \\
\text { children who } \\
\text { participated in } \\
\text { motocross }\end{array}$ & $\begin{array}{l}18 \% \text { LOC with } 8 \text { and } \\
5 \text { abnormal Glasgow } \\
\text { Coma Scores and CT }\end{array}$ \\
\hline
\end{tabular}


Table I. Continues

\begin{tabular}{|c|c|c|c|c|c|c|}
\hline Study & Design & $\begin{array}{l}\text { Study } \\
\text { Period }\end{array}$ & Sample (n) & $\begin{array}{l}\text { Gender } \\
(\mathrm{M})\end{array}$ & $\begin{array}{l}\text { Aim/Purpose of } \\
\text { Study }\end{array}$ & Rate of Injuries \\
\hline $\begin{array}{l}\text { Miller et } \\
\text { al., } 2016\end{array}$ & $\begin{array}{l}\text { Prospective } \\
\text { Cohort }\end{array}$ & $\mathrm{NR}$ & $\mathrm{n}=782$ individuals & $85 \%$ & $\begin{array}{l}\text { Assess concussion } \\
\text { knowledge among } \\
\text { amateur motocross } \\
\text { riders }\end{array}$ & $\begin{array}{l}75.1 \%(\mathrm{n}=587) \text { with } \\
\text { prior concussion history }\end{array}$ \\
\hline $\begin{array}{l}\text { Daniels et } \\
\text { al., } 2015\end{array}$ & $\begin{array}{l}\text { Retrospective } \\
\text { population- } \\
\text { based cohort }\end{array}$ & $2000-2007$ & $\begin{array}{l}\mathrm{n}=298 \text { accidents }(\mathrm{n}=248 \\
\text { patients })\end{array}$ & $93.1 \%$ & $\begin{array}{l}\text { Confirm the rate } \\
\text { of head and spine } \\
\text { injuries in the } \\
\text { pediatric population } \\
\text { following motocross } \\
\text { accidents }\end{array}$ & $\begin{array}{l}61.2 \% \text { occurred at } \\
\text { a formal motocross } \\
\text { course; } \\
20 \% \text { Head injuries/ } \\
\text { TBIs; } 95 \% \text { LOC }\end{array}$ \\
\hline
\end{tabular}

As opposed to land-based sports such as snowboarding or mountain biking, during water-sports (e.g. windsurfing), concussions may be caused by a strike from a board, a boom or even by falls $(31,32)$. While considering watersports, concussion accounted for $4.2-5.9 \%$ of surfing-related injuries (33). Another study found that concussion accounted for $8.2 \%$ of surfing injuries (9). Moreover, $5.9 \%$ of kitesurfing related injuries and between $3.9-13 \%$ of personal watercraft riding related injuries were concussions (33). It is important to note that this study, as with many other studies that report on injuries in extreme sports athletes, used the term "head injury" to include concussion, TBI, lacerations, skull and facial fractures, contusions etc. As there are vast differences in these injuries and how they are treated clinically, it is important from an epidemiological standpoint to distinguish between these types in further research. This remarks the current lack of data that reports on the incidence of concussions alone in many of these extreme sports. The majority of papers in extreme sports are focused around winter-based activities, like snowboarding or skiing (9); motorized vehicle sports, like All-Terrain Vehicle (ATV) competitions and motocross (23-25,34); and skateboarding $(9,27)$. One paper observing concussions in hang-gliding reported that concussions accounted for $18.7 \%$ of hang-gliding accidents (35). As for another extreme air sport, BASE jumping, the only paper identified mentioned concussion as a "common injury", along with ankle sprains and fractures and bruised knees (26). Precise data for most other extreme sports are still missing, which may be due to their relatively new classification as extreme or action sports.

\section{Initial diagnosis and symptoms of concussion}

Appropriate identification and management of concussion from the initial onset are critical for optimal care and reduction of persistent post-concussive symptoms (9). Given the high rate of concussion in several extreme sports, a plan to have on-site concussion evaluation should be implemented when developing event-side medical coverage (36). As previously discussed, diagnosing a concussion can be challenging and is often controversial, due to the lack of precise biomarkers (e.g. no blood test or imaging study) to confirm the diagnosis. As such, inclusion of concussion trained medical providers on-site during extreme sport competitions is warranted. As initial presentation varies significantly among athletes, recognition of the many signs and symptoms of a concussion should be performed by trained medical personnel to determine if the injury involves loss of consciousness (LOC) or altered mental status (37).

According to the American Academy of Pediatrics only 10\% of patients with concussions experience a loss of consciousness (24). Despite this, a study performed by Daniels et al. observed that $95 \%$ of patients who suffered a concussion after a motocross accident had a positive LOC (24). Another study found that there was a high rate $(27.1 \%)$ of LOC at the scene for head injuries among skaters (27). This study also found that LOC at the scene accounted for $14.6 \%$ of airborne and $16.7 \%$ of climbing head injuries; however, it was not explicitly stated that these LOC incidents were the result of a concussion or more severe TBI (27). This may suggest an abnormally high rate of LOC concurrent with concussion relative to other published values among traditional sport athletes (e.g. football); for reference, one study found that $9 \%$ of all football concussions resulted in a loss of consciousness (38). The percentage of concussions presenting with LOC may be higher in extreme sports, because of the higher magnitude of forces and accelerations that the head may be exposed to relative to other sports, although no studies have directly investigated this idea. Although conceivable that the high speeds and forceful impacts of extreme sports lead to higher incidence of LOC, it is also plausible that an injury resulting from a LOC causes more 
concern leading to a higher incidence of emergency department (ED) visits. In contrast, a seemingly more apparently minor injury (one that does not involve LOC) may not result in a visit to a healthcare provider, particularly if there is no medical oversight present at the competition.

In general, the most common presentation of a concussion is headache with secondary dizziness (39). Initial dizziness at presentation is associated with 6.4 times higher risk relative to any other initial symptom in predicting a protracted recovery (40); although, many different elements may contribute to development of persistent post-concussion symptoms. Initial symptoms can also include amnesia (retrograde and posttraumatic), nausea, fatigue, fogginess, blurry vision, phonophobia, photophobia, difficulty concentrating, and increased emotionality $(5,6)$. Concussion symptoms have been subdivided into groups including headache/migraine, vestibular, ocular, cognitive, mood/anxiety and cervical (37). Returning to extreme sports without complete recovery, shown by the absence of post-concussion symptoms, could place the athlete at more significant risk for another concussion. Due to the increased height, forces and speed of many of these extreme sports, lack of precise concentration, increased anxiety, balance/coordination impairment, inability to make last minute decisions and/or slowed reaction time could result in severe consequences as compared to more traditional sports.

Perhaps as a result of the difficulty deciphering the diagnosis among the possible presentable symptoms, many professional, collegiate, and high school organizations use time-sensitive concussion procedures for use on the sidelines or in clinical evaluations during or shortly after traditional sport practices or competition. One such program used for concussion evaluation is the Sideline Concussion Assessment Tool $-5^{\text {th }}$ Edition (SCAT5), a standardized tool for evaluating concussions designed for use by physicians and licensed healthcare professionals, which incorporates immediate on-field assessments, off-field assessment, cognitive and neurological screening. Although not required, the SCAT5 can also incorporate valuable, pre-season baseline testing for athletes in the traditional sport settings to compare symptom scores and track overall improvement as they progress through the standard return to play (RTP) protocol (41). The initial assessment for concussions, which includes athlete and injury history in conjunction with symptom questionnaires and functional tests, are critical in determining concussions from other types of head injuries. Unlike most traditional sports, many extreme sports lack formal baseline evaluation procedures such as the SCAT5. In this event, comparing post-injury performance to established normative values may be useful given that this approach has similar sensitive and specificity compared to baseline evalu- ation $(42,43)$. Extreme sports athletes may possess different characteristics than traditional sport athletes, yet, caution should still be used when interpreting post-injury performance among this cohort of individuals.

A formal concussion diagnosis would ideally be made by a healthcare provider, who is familiar with the athlete and an expert in the recognition and evaluation of concussions. Graded symptom checklists provide a more objective tool for assessing initial and prolonged symptoms (39). Standardized and updated sideline assessment tools (e.g. SCAT5) provide a helpful guide for the evaluation of head injuries, but contrary to organized sports, most practice sessions and participation in extreme sports are void of any sideline healthcare provider, leading to inconsistency in the assessment of head injuries. In most cases, participants are required to self-report their injuries and symptoms to their primary care provider, local urgent care or emergency department or to a sports medicine physician. Symptoms are also often underreported commonly through these channels for extreme sport athletes, creating an additional challenge for the healthcare provider. However, this phenomenon is not solely found in extreme sport athletes, but can be found also in traditional sports, where athletes may underreport their injuries due to pressure to perform from coaches, teammates or parents (7).

In a concussion, imaging is reserved for athletes where intracerebral bleeding is suspected, as conventional brain imaging methods lack the sensitivity to detect the subtle changes that may be part of the pathophysiology of concussion (44). However, among children admitted to a trauma center in Pennsylvania with a diagnosis of mTBI or concussion, a CT scan proved more likely to find abnormalities in those trauma related to high speed sport activities $(27 \%$; $=14)$, such as snowboarding, skiing, skateboarding and motocross riding, than to court sport activities $(10 \% ; n=2)(45)$. This finding suggests that physicians who treat extreme sports athletes who have sustained a likely concussion should always consider the possibility of more severe injuries resulting from high energy trauma and CT imaging should be used to rule out hemorrhage or even traumatic carotid dissection potentially resulting in brain ischemia (46).

\section{Persistent post-concussion symptoms \& management}

Numerous patients, in both traditional and extreme sports, do not receive the diagnosis soon after onset, resulting in poor management that may be improper, potentially leading to negative outcomes such as altered quality of life (46). As a result, without early care and proper treatment, these athletes may be at an increased risk of persistent 
post-concussion symptoms (PPCS). However, currently we are unaware of any studies to this point that have directly addressed this question among this population (47). PPCS is defined as the presence of concussion-related symptoms that began at the time of injury and do not recover within one month of injury. When considering athletes, it is essential to support the psychological piece that sports play in their identity construct and the external pressure they may feel related to performance, while supporting them in the return to sport process (7). The associated symptoms that may persist during PPCS include depression, chronic fatigue, visually induced dizziness, headache, fatigue, sleep disturbance, vertigo, irritability, anxiety, apathy and cognitive slowness, or difficulties in exercising (7). The biggest clinical challenge is to determine whether prolonged symptoms are a true reflection of PPCS or a secondary manifestation of premorbid clinical depression or migraine, which are commonly concurrent conditions.

\section{Treatment}

Concussion management of extreme sports athletes should align with the most up-to-date treatment of concussed athletes in traditional sports regarding initial management of symptoms, optimizing nutrition, hydration and sleep, managing return to learn/work accommodations, early implementation of controlled aerobic activity, and rehabilitation of vestibular, ocular and cervical injury. Currently, athletes affected by concussion are encouraged to follow the most recent RTP guidelines that were designed for the use in traditional sports as directed by their physician (4). Specific RTP protocols for various extreme sports should be developed to aid the athlete in the process of gradual return to sport. As an example, for mountain bike athletes, Step 1 may include 20 minutes of light to moderate spinning on a stationary bike/trainer, Step 2 may include 45 minutes of moderate to hard cycling on a flat surface on bike trails or road riding, Step 3 may increase to a 1-1.5-hour team practice of moderate mountain bike riding on trails, Step 4 may progress to a full 2-hour team practice on trails without restrictions and Step 5 return to full mountain bike competitions. Patients with PPCS may need a multidisciplinary rehabilitation protocol. Rehabilitation should include a variety of aspects, from vestibular and cervical spinal, to cognitive and autonomic (exercising - aerobically training) (3). In addition to the standard RTP protocol for traditional athletes, there is also ongoing research on the potential benefits of sensorimotor and neuromuscular training for injury prevention in patients reporting post-concussive symptoms. Concussions commonly cause functional disturbances of sensorimotor control that are crucial for athletes as they process a variety of visual and proprioceptive cues during sport participation. In a recent pilot study performed on collegiate soccer players, significant improvements were noted in "static balance, cervical flexor neuromotor control/endurance, and near-point convergence", as well as a decrease in athlete injury exposure after the implementation of eight sensorimotor training programs (48). Additionally, there is evidence that similar cervicovestibular rehabilitation, as well as treatment of concurrent whiplash associated disorder, may be essential in treating those categorized with protracted symptoms in both extreme sport and traditional sport athletes $(10,49)$. Although these studies show the promise of such programs to act as both preventative measures and rehabilitation programs, more research is needed to solidify their utility in both traditional sport and extreme sport athletes.

\section{Prevention}

As in traditional sports like hockey, football, and men's lacrosse, the use of helmets in extreme sports has been encouraged and utilized as an injury prevention tool. Sulheim et al. (46), found that among alpine skiers and snowboarders, helmet use reduced the chance of head injuries by $60 \%$; however, it was not specified whether these head injuries were skull fractures, severe TBIs, or concussions (24). A similar meta-analysis on concussion reduction strategies in sport found that there is a protective effect of helmets in skiing and snowboarding for head injuries, but also lacked definition of whether or not these head injuries were concussions (50). In a motocross study, $71.7 \%$ of the reported head-injury cases had confirmed helmet use (24). Although the use of helmets and protective gear may be insufficient to avoid a concussion in all extreme sports, it may reduce the severity of a head injury, which remains of great importance (51).

The use of protective headgear varies among extreme sports athletes, depending on the sport in which they participate. Headgear is rarely worn by certain extreme sports participants (e.g. surfing, skateboarding) due to the perception that it is unnecessary, may distract from the completion of certain skills required by the sport, and lack of evidence of its benefit (52). Contrastingly, helmet use is common among snowboarders, skiers and motocross athletes. Furthermore, some extreme sports, like motocross and kiteboarding, have helmet requirements depending on what geographical region the sport participation occurs (25). For example, the International Kiteboarding Association (IKA) implemented the mandatory use of helmets of at least 300 square centimeters in 2019 (53). Moreover, in a retrospective study reported by Daniels et al., all patients who presented to the emer- 
gency department with an injury received while riding at a formal motocross course were helmeted (24). This shows the willingness of extreme sports athletes, at least in motocross, to abide by regulatory rules, if implemented.

In addition to preventative equipment, environmental variables are a factor related to injury risk reduction that relate to the extreme sports athlete more than other sports (e.g. basketball). For instance, in alpine skiing and snowboarding, the risk of concussion is 2.5 times higher on rough or ungroomed snow than in soft snow (54). Moreover, concussions and head/face injuries are more common on terrain parks with the specialized jumps and ramps than other slopes (55-57). This may signify a future direction in concussion prevention measures in extreme sports that may be based on creating standardized environmental conditions for participation. Similarly, in surfing, a correlation between increased surfing competence and the incidence of head lacerations and skull fractures has been reported- most likely due to the fact that an expert surfer prefers more difficult conditions over shallow reefs while a beginner will generally surf smaller breaks (58). Further research is needed to explore this association before protective measures can be made in competition for varying age-ranges and skill level.

Contrastingly, the National Center for Injury Prevention and Control reports that children may be at a greater risk in these extreme conditions for injury occurrence because of immature or underdeveloped coordination, skills and perception and because of reduced emotional maturity and judgment compared to adults, especially in the presence of peers (18). Sharma et al. also found that teens and young adults accounted for the highest percentage of extreme sports injuries in their 12 year review of National Electronic Injury Surveillance System data for seven popular extreme sports featured at the Winter and Summer X Games for head and neck injuries (9). It is valuable for all health care providers to be aware of these risks so they can adequately educate families and coaches of risk among extreme sports athletes of varying ages and skill levels.

Additionally, a study done at a motocross race event showed approximately half of racers reported suffering concus-

\section{REFERENCES}

1. Wing BH, Tucker BJ, Fong AK, Allen MD. Developing the Standard of Care for Post-Concussion Treatment: Neuroimaging-Guided Rehabilitation of Neurovascular Coupling. Open Neuroimaging J. 2017;11:58-71.

2. Tator CH. Let's Standardize the Definition of Concussion and Get Reliable Incidence Data. Can J Neurol Sci. 2009 Jul;36(4):405-6. sion-related symptoms from the earlier season, while a third reported multiple concussive incidents, but continued to participate in the event (23). This continued participation immediately after a concussive event is alarming due to the elevated prospect of developing persistent post-concussive symptoms or second impact syndrome if another head impact occurs soon after the primary impact. In the same study, authors observed considerable misconceptions and lack of symptom knowledge in amateur extreme sport athletes compared to their traditional sport peers that often undergo mandatory education through centralized schoolbased administration (23). As a result, the benefits of formal education of athletes and their families as a preventative measure for concussions should be explored.

\section{CONCLUSIONS}

With this brief review, we aimed to emphasize the need to document and record mild traumatic brain injuries in extreme sports as well as to need to develop a RTP guideline for healthcare professionals dealing with extreme sports athletes. Furthermore, medical coverage with personnel trained in concussion management remains a key element during extreme sports competitions to ensure early injury recognition and proper management and referral. Secondly, we have highlighted the importance of prevention and multidisciplinary management. Athletes may be developing a variety of symptoms and only a dynamic therapeutic approach, which will consider the different component of concussion, will be able to ease their symptoms and reconstitute normal brain function. In the future, advanced neuroimaging techniques such as functional magnetic resonance imaging (fMRI), may help to better understand the pathophysiology of concussion and the effects of interventions in concussed athletes on a clinical basis.

\section{CONFLICT OF INTERESTS}

The authors declare that they have no conflict of interests (59).

3. Feddermann-Demont N, Echemendia RJ, Schneider KJ, Solomon GS, Hayden KA, Turner M, et al. What domains of clinical function should be assessed after sport-related concussion? A systematic review. Br J Sports Med. 2017 Jun;51(11):903-18.

4. McCrory P, Meeuwisse WH, Aubry M, Cantu B, Dvorák J, Echemendia RJ, et al. Consensus statement on concussion in sport: the 4th International Conference on Concussion in 
Sport held in Zurich, November 2012. Br J Sports Med. 2013 Apr;47(5):250-8.

5. Iverson GL, Gardner AJ, Terry DP, Ponsford JL, Sills AK, Broshek DK, et al. Predictors of clinical recovery from concussion: a systematic review. Br J Sports Med. 2017 Jun;51(12):941-8.

6. Zemek R, Barrowman N, Freedman SB, Gravel J, Gagnon I, McGahern C, et al. Clinical Risk Score for Persistent Postconcussion Symptoms Among Children With Acute Concussion in the ED. JAMA. 2016 Mar 8;315(10):1014-25.

7. Leddy JJ, Sandhu H, Sodhi V, Baker JG, Willer B. Rehabilitation of Concussion and Post-concussion Syndrome. Sports Health. 2012 Mar;4(2):147-54.

8. Meehan WP, Mannix RC, O’Brien MJ, Collins MW. The prevalence of undiagnosed concussions in athletes. Clin J Sport Med Off J Can Acad Sport Med. 2013 Sep;23(5):339-42.

9. Sharma VK, Rango J, Connaughton AJ, Lombardo DJ, Sabesan VJ. The Current State of Head and Neck Injuries in Extreme Sports. Orthop J Sports Med. 2015 Jan;3(1):2325967114564358.

10. Henjum M, Mattson C, Feletti F. Rehabilitation of Extreme Sports Injuries. In: Extreme Sports Medicine. Feletti F. Springer International Publishing Switzerland; 2016. p. 429-49.

11. Buckley RC. To Analyze Thrill, Define Extreme Sports. Front Psychol. 2018;9:1216.

12. Thorpe H. Action Sports, Social Media, and New Technologies: Towards a Research Agenda. Commun Sport. 2017 Oct 1;5(5):554-78.

13. Coakley J. Youth Sports: What Counts as "Positive Development?" J Sport Soc Issues. 2011 Aug 1;35(3):306-24.

14. Brymer E, Feletti F. Beyond risk: the importance of adventure in the everyday life of young people. Ann Leis Res. 2019 Aug 30;0(0):1-18.

15. Gosteli G, Yersin B, Mabire C, Pasquier M, Albrecht R, Carron P-N. Retrospective analysis of 616 air-rescue trauma cases related to the practice of extreme sports. Injury. 2016 Jul;47(7):1414-20.

16. Feletti F. Extreme Sports Medicine. Springer; 2016. 456 p.

17. Pain MTG, Pain MA. Essay: Risk taking in sport. Lancet Lond Engl. 2005 Dec;366 Suppl 1: S33-34.

18. Caine DJ, Provance AJ. Pediatric and adolescent injury in adventure and extreme sports. Res Sports Med. 2018 Apr 26;26(sup1):5-19.

19. Davids K, Brymer E, Seifert L, Orth D. A Constraints-Based Approach to the Acquisition of Expertise in Outdoor Adventure Sports. In 2014.

20. Immonen T, Brymer E, Orth D, Davids K, Feletti F, Liukkonen J, et al. Understanding Action and Adventure Sports Participation-An Ecological Dynamics Perspective. Sports Med - Open. 2017 Dec;3(1):18.

21. Cohen R, Baluch B, Duffy LJ. Defining Extreme Sport: Conceptions and Misconceptions. Front Psychol. 2018;9:1974.

22. Immonen T, Brymer E, Davids K, Liukkonen J, Jaakkola T. An Ecological Conceptualization of Extreme Sports. Front Psychol. 2018;9:1274.

23. Miller KO, Langdon JL, Burdette GP, Buckley TA. Concussion knowledge among amateur motocross riders. Concussion. $2016 \mathrm{Jul}$ 6;1(3):CNC16.
24. Daniels DJ, Clarke MJ, Puffer R, Luo TD, McIntosh AL, Wetjen NM. High occurrence of head and spine injuries in the pediatric population following motocross accidents. J Neurosurg Pediatr. 2015 Mar 1;15(3):261-5.

25. Larson A, Stans A, Shaughnessy W, Dekutoski M, Quinn M, McIntosh A. Motocross Morbidity: Economic Cost and Injury Distribution in Children. J Pediatr Orthop. 2009 Dec;29(8):847-50.

26. Soreide K, Ellingsen C, Knutson V. How Dangerous is BASE Jumping? An Analysis of Adverse Events in 20,850 Jumps From the Kjerag Massif, Norway. J Trauma Inj Infect Crit Care. 2007 May;62(5):1113-7.

27. Weber CD, Horst K, Nguyen AR, Lefering R, Pape H-C, Hildebrand F, et al. Evaluation of severe and fatal injuries in extreme and contact sports: an international multicenter analysis. Arch Orthop Trauma Surg. 2018 1;138(7):963-70.

28. Nakaguchi H, Fujimaki T, Ueki K, Takahashi M, Yoshida H, Kirino T. Snowboard head injury: prospective study in Chino, Nagano, for two seasons from 1995 to 1997. J Trauma 1999 Jun;46(6):1066-9.

29. Bridges EJ, Rouah F, Johnston KM. Snowblading injuries in Eastern Canada. Br J Sports Med. 2003 Dec;37(6):511-5.

30. Machold W, Kwasny O, Gässler P, Kolonja A, Reddy B, Bauer E, et al. Risk of injury through snowboarding. J Trauma. 2000 Jun;48(6):1109-14.

31. Jablecki CK, Garner S. Neurological complications of windsurfing (sailboarding). Semin Neurol. 2000;20(2):219-23.

32. Kalogeromitros A, Tsangaris H, Bilalis D, Karabinis A. Severe accidents due to windsurfing in the Aegean Sea. Eur J Emerg Med Off J Eur Soc Emerg Med. 2002 Jun;9(2):149-54.

33. Pikora TJ, Braham R, Mills C. The epidemiology of injury among surfers, kite surfers and personal watercraft riders: wind and waves. Med Sport Sci. 2012;58:80-97.

34. Moroney P, Doyle M, Mealy K. All-terrain vehicles--unstable, unsafe and unregulated. A prospective study of ATV-related trauma in rural Ireland. Injury. 2003 Mar;34(3):203-5.

35. Margreiter R, Lugger LJ. Hang-gliding accidents. Br Med J. 1978 Feb 18;1(6110):400-2.

36. Feletti F. Extreme Sports Medicine. p. 139-150: Springer; 2016. 456 p.

37. Doperak J, Anderson K, Collins M, Emami K. Sport-Related Concussion Evaluation and Management. Clin Sports Med. 2019 Oct;38(4):497-511.

38. Guskiewicz KM, Weaver NL, Padua DA, Garrett WE. Epidemiology of concussion in collegiate and high school football players. Am J Sports Med. 2000 Oct;28(5):643-50.

39. Harmon KG, Drezner J, Gammons M, Guskiewicz K, Halstead M, Herring S, et al. American Medical Society for Sports Medicine Position Statement: Concussion in Sport. Clin J Sport Med. 2013 Jan;23(1):1.

40. Mucha A, Collins MW, Elbin RJ, Furman JM, Troutman-Enseki C, DeWolf RM, et al. A Brief Vestibular/Ocular Motor Screening (VOMS) assessment to evaluate concussions: preliminary findings. Am J Sports Med. 2014 Oct;42(10):2479-86.

41. Baugh CM, Kroshus E, Stamm JM, Daneshvar DH, Pepin MJ, Meehan WP. Clinical Practices in Collegiate Concussion Management. Am J Sports Med. 2016 Jun;44(6):1391-9.

42. Chin EY, Nelson LD, Barr WB, McCrory P, McCrea MA. Reliability and Validity of the Sport Concussion Assessment 
Tool-3 (SCAT3) in High School and Collegiate Athletes. Am J Sports Med. 2016 Sep;44(9):2276-85.

43. Hänninen T, Parkkari J, Tuominen M, Öhman J, Howell DR, Iverson GL, et al. Sport Concussion Assessment Tool: Interpreting day-of-injury scores in professional ice hockey players. J Sci Med Sport. 2018 Aug;21(8):794-9.

44. Slobounov S, Gay M, Johnson B, Zhang K. Concussion in athletics: ongoing clinical and brain imaging research controversies. Brain Imaging Behav. 2012 Jun;6(2):224-43.

45. Bramley H, Mcfarland C, Lewis MM, Shaffer ML, Cilley R, Engbrecht B, et al. Short-term outcomes of sport- and recreation-related concussion in patients admitted to a pediatric trauma service. Clin Pediatr (Phila). 2014 Jul;53(8):784-90.

46. Spanjersberg WR, Schipper IB. Kitesurfing: when fun turns to trauma-the dangers of a new extreme sport. J Trauma. 2007 Sep;63(3):E76-80.

47. Eisenberg MA, Meehan WP, Mannix R. Duration and course of post-concussive symptoms. Pediatrics. 2014 Jun;133(6):999-1006.

48. Reneker JC, Babl R, Pannell WC, Adah F, Flowers MM, Curbow-Wilcox K, et al. Sensorimotor training for injury prevention in collegiate soccer players: An experimental study. Phys Ther Sport Off J Assoc Chart Physiother Sports Med. 2019 Sep 27;40:184-92.

49. Schneider KJ, Meeuwisse WH, Nettel-Aguirre A, Barlow K, Boyd L, Kang J, et al. Cervicovestibular rehabilitation in sport-related concussion: a randomised controlled trial. Br J Sports Med. 2014 Sep;48(17):1294-8.

50. Emery CA, Black AM, Kolstad A, Martinez G, Nettel-Aguirre A, Engebretsen L, et al. What strategies can be used to effectively reduce the risk of concussion in sport? A systematic review. Br J Sports Med. 2017 Jun;51(12):978-84.
51. Sulheim S, Holme I, Ekeland A, Bahr R. Helmet Use and Risk of Head Injuries in Alpine Skiers and Snowboarders. JAMA. 2006 Feb 22;295(8):919-24.

52. Taylor DM, Bennett D, Carter M, Garewal D, Finch C. Perceptions of surfboard riders regarding the need for protective headgear. Wilderness Environ Med. 2005;16(2):75-80.

53. Babini M. Welcome to the International Kiteboarding Association [Internet]. International Kiteboarding Association. 2019 [cited 2019 Oct 21]. Available from: http://internationalkiteboarding.org/.

54. Ackery A, Hagel BE, Provvidenza C, Tator CH. An international review of head and spinal cord injuries in alpine skiing and snowboarding. Inj Prev. 2007 Dec;13(6):368-75.

55. Brooks MA, Evans MD, Rivara FP. Evaluation of skiing and snowboarding injuries sustained in terrain parks versus traditional slopes. Inj Prev J Int Soc Child Adolesc Inj Prev. 2010 Apr;16(2):119-22.

56. Russell K, Meeuwisse W, Nettel-Aguirre A, Emery CA, Wishart J, Romanow NTR, et al. Characteristics of injuries sustained by snowboarders in a terrain park. Clin J Sport Med Off J Can Acad Sport Med. 2013 May;23(3):172-7.

57. Goulet C, Hagel B, Hamel D, Légaré G. Risk factors associated with serious ski patrol-reported injuries sustained by skiers and snowboarders in snow-parks and on other slopes. Can J Public Health Rev Can Sante Publique. 2007 Oct;98(5):402-6.

58. Lowdon BJ, Pateman NA, Pitman AJ. Surfboard-riding injuries. Med J Aust. 1983 Dec 10;2(12):613-6.

59. Padulo J, Oliva F, Frizziero A, Maffulli N. Muscles, Ligaments and Tendons Journal - Basic principles and recommendations in clinical and field Science Research: 2018 update. MLTJ 2018; 8(3): $305-307$. 